Análisis discursivo de las vacunas anticovid-19 en Twitter

Karines Rodríguez; Yamile Haber Guerra; Rafael Ángel Fonseca Valido

Question/Cuestión, Nro.70, Vol.3, diciembre 2021

ISSN: 1669-6581

URL de la Revista: https://perio.unlp.edu.ar/ojs/index.php/question/

IICom -FPyCS -UNLP

DOI: https//doi.org/10.24215/16696581e624

\title{
Análisis discursivo de las vacunas anticovid-19 en Twitter
}

Discursive analysis of anticovid-19 vaccines on Twitter

Karines Rodríguez

Universidad de Oriente

Cuba

karines1991@gmail.com

https://orcid.org/0000-0002-9349-9386

Yamile Haber Guerra

Cuba

yhaber@uo.edu.cu

https://orcid.org/0000-0001-7200-5714

Rafael Ángel Fonseca Valido

Cuba 


\section{Resumen}

El presente informe expone los resultados de un estudio de caso descriptivo asociado a los tweets publicados bajo los hashtags \#vacunacoronavirus y \#coronavirusvaccine. Un aporte fundamental de la investigación es la propuesta de una metodología de análisis del discurso asistido por minería de datos en un escenario asociado a mensajes multisoportes. El presente artículo con un enfoque mixto fue aplicado sobre una muestra de 1 millón de tweets extraídos de manera aleatoria por las aplicaciones Stela y Brand24. Mostramos la necesaria interrelación metodológica del uso de softwares y herramientas cuantitativas, así como la perspectiva cualitativa para determinar la interpretación de la representación simbólica que se emitió sobre la vacunación anti-covid 19. Los resultados presentan los encuadres, la polaridad, y las percepciones que adoptan los usuarios respecto a vacunas de comercialización internacional.

Palabras clave: minería de datos; análisis del discurso; representación simbólica; vacuna; coronavirus

\section{Abstract}

This report presents the results of a descriptive case study associated with tweets published under the hashtags \#vaccunacoronavirus and \#coronavirusvaccine. A fundamental contribution of the research is the proposal of a methodology of discourse analysis assisted by data mining in a scenario associated with multi-supported messages. The present paper with a mixed approach was applied on a sample of 1 million tweets randomly extracted by Stela and Brand24 applications. We show the necessary methodological interrelation of the use of software and quantitative tools, as well as the qualitative perspective to determine the interpretation of the symbolic representation that was issued on anti-covid 19 vaccination. The results present the framing, polarity, and perceptions adopted by users regarding internationally marketed vaccines. 
Keywords: data mining; discourse analysis; symbolic representation; vaccine; coronavirus.

\section{Introducción}

El principal cambio en el estudio del discurso con la irrupción de las redes sociales es la adecuación de modelos metodológicos al estilo de producción, distribución, interpretación y consumo de los mensajes en el escenario actual de la web. Ya no nos comunicamos por una computadora, sino con la cultura codificada en forma digital (Manovich, 2002).

Las plataformas de Internet y sus prácticas comunicativas permiten cambios epistemológicos centrales atribuibles a la naturaleza del objeto u objetos de estudio que la irrupción de las tecnologías digitales impone a la semiótica (Pérez, 2013). Twitter es una de las redes sociales más usadas en la web, con 353 millones de usuarios (Hootsuite \& We are social, 2021), según Engesser y Humpretch (2015) es el espacio en el cual periodistas y políticos son especialmente activos. Numerosos autores han valorado el potencial de Twitter como espacio para la comunicación (Gómez, 2014; Moya y Herrera, 2015; Pérez et al, 2017; Pérez, 2019). Se trata de una herramienta utilizada por los actores y la audiencia de la vida política y noticiosa para la conversación sobre tendencias y sucesos.

Las investigaciones científicas en los espacios de redes sociales han utilizado mayormente el escenario de microblogging puesto que permite acceder a sus bases de datos y descargar la información, de manera más fácil que otra red social, sin riesgo de transgresión ética o de la propiedad intelectual (Pérez, 2017: 13), y sin seguimiento obligatorio in situ al objeto de estudio, así mismo es la plataforma en la que se han desarrollado más softwares abiertos para la extracción y filtrado de resultados. Así mismo ha sido una de las fuentes principales para la revisión del procesamiento del lenguaje natural en la red.

No obstante, los apuntes metodológicos de las investigaciones de los últimos años en el microblogging son muy variables. Los autores definen propuestas teórico metodológicas poco concluyentes en tanto la variedad de formatos, prácticas comunicativas y estilo de los textos en Twitter. En los textos científicos asociados al análisis del discurso ha prevalecido el análisis del texto escrito, probablemente porque las metodologías y los softwares asociados a este soporte son más concluyentes con respecto a otros formatos. Así por ejemplo las técnicas más empleadas en relación son la lingüística de corpus, el análisis de sentimientos y la detección de las polaridades que suelen vincularse con aspectos teóricos más analógicos 
como la lingüística sistémico funcional (Zappavingia, 2011), la retórica (Gutiérrez, 2016; Dackow, 2020), o la argumentación (Padilla, 2015; Ventura, 2016, Alonso, 2016). Algunos ejemplos están más centrados en la posibilidad de análisis que ofrece la computación y los softwares, algunos casos significativos son los de Arcila et al. (2017), Verbeke et al. (2017) y Van Hee (2017).

Otros investigadores no renuncian a las posibilidades del análisis cualitativo en Twitter (Gómez, 2014; Padilla, 2015; Ventura, 2016; Gutiérrez, 2016; Marín y Quintero, 2018; Pérez, 2019; Olave, 2019; Dackow, 2020, Méndez, 2020) sobre todo en los que se relacionan con otros soportes como fotos y videos (Nuñez y Fernández, 2018; Sharag et al., 2018; Ventura, 2018; Mancera, 2020; Molpeceres y Filardo, 2020; Ozduzen y Macgarry, 2020; Du et al., 2020) cuyo análisis simbólico es solo permisible para el investigador dado que la capacidad de la inteligencia artificial no se ha desarrollado ni es efectiva completamente en las conclusiones respecto a estos formatos. En consecuencia, abundan mayoritariamente los enfoques asociados a Twitter y el text mining (Reyes, 2012; Ortega et al, 2013; Vilares et al., 2013; Abascal et al, 2014; Almgrem y Olssom, 2015; Vilariño et al, 2015; Arcila et al, 2017, Figueira, y Guimarães, 2017; Hernández, 2017; Kwabla at al, 2017; Martínez, 2017; Reyes at al., 2017; González at al, 2018; García et al, 2019; Ferreira, 2019; Henríquez et al., 2019)

Percastre et al. (2017) proponen un modelo denominado Top discussion indicator (TDI) o Indicador de la máxima discusión. Su objetivo es ayudar a los investigadores a obtener un conjunto representativo de un universo que, aunque pueda superar cientos de miles de registros, incluya la mínima información que permita generalizar los resultados basados en uno o varios indicadores cuantificables (o categorías contextuales).

Siguiendo la propuesta esta investigación tendremos en cuenta al texto rey como el cuerpo del tweet mientras que como parte del mensaje identificaremos un paratexto que se asume como el contenido complementario asociado al texto rey y que forma parte de su significación para representar el sentido, esto es las imágenes, encuestas, videos y otros multisoportes añadidos, así como los valores que añaden otros usuarios al tweet: retweets y me gusta . Así mismo asumiremos como cotexto las opciones de respuestas a dicho texto que, aunque parten de otro autor, son dependientes de la afirmación iniciada en el texto rey.

La relación entre diseminación de información en Twitter y estados de emergencia ocasionados por enfermedades ha sido estudiada en los últimos años en referencia 
principalmente al virus del Ébola y a la influenza A H1N1, desde el campo de la interrelación entre salud y estudios de medios (Percastre et al., 2019; Towers et al, 2015). Mientras Sued y Cebral (2020) en un contexto más reciente realizan un análisis de sentimientos y una extracción de términos asociados a emociones en una relación de figuras públicas, lenguaje y autoridad que logra complementar la relación discursiva con la relación social en Twitter respecto a las voces autorizadas en Twitter durante la pandemia de COVID 19.

Algunos estudios más recientes relacionan estrategias mixtas de uso metodológico mostrando una necesaria vinculación entre el análisis de contenido, el análisis de discurso y la minería de datos que contiene los dos primeros como técnicas afines con apoyo de softwares de extracción y filtrado. Así por ejemplo Vela et al. (2021) combinan técnicas de análisis del social media mining con estrategias cualitativas propias del análisis lingüístico, aunque desde el punto de vista teórico, se apoya en conceptos de las teorías del encuadre y de la valoración y asumen una muestra basada en texto escrito asociada a la etiqueta \#mequedoencasa ante la emergencia producida por covid 19. Pulido et al. (2021) además expone el uso institucional de Twitter para combatir la infodemia causada por la crisis sanitaria de la Covid-19 aunque utiliza una metodología de corte cuantitativo.

Pocos casos de estudio (Gindin et al., 2019; Rodríguez y Haber, 2020; Rodríguez, Haber y Gómez, 2021) relacionan las palabras, los hashtags y las imágenes en un acercamiento de tipo semiótico al mensaje, algo que también hacen Rebollo y Gualda (año) en el análisis de la imagen proyectada en Twitter sobre los refugiados y Pérez y García (2018). Sin embargo, en la mayoría de las investigaciones en Twitter se fragmenta el estudio del mensaje atendiendo a su soporte, es decir, o se enmarca la investigación en el texto escrito o en la imagen lo que supone un análisis incompleto de la producción simbólica. Por lo tanto, exponemos un criterio de análisis mixto que relaciona el análisis cuantitativo y el cualitativo, que, además de incluir el uso de softwares para detección y filtrado tan efectivos en las dinámicas de investigación contemporáneas, propone una perspectiva multisoporte.

\section{Análisis de discurso en Twitter}

Las noticias deben estudiarse como una forma de discurso público y como un proceso de interacción social (Van Dijk, 2000: 26). Es importante entonces el contexto y las relaciones sociales que deriva.Así por ejemplo la confluencia de archivos multisoporte dado que un 
mensaje en Internet puede darse a través de una representación verbal y otra semiótica (imágenes, videos, audios, etc). Barthes (1977), en su preocupación por una lingüística del discurso y por la semiótica, describe las relaciones entre texto e imagen como de tres tipos: anclaje, el texto permite dilucidar la imagen, ilustración, la imagen permite aclarar el texto y relevo: tanto el texto como la imagen están a un mismo nivel. Estas relaciones entre texto y producción icónica también se producen en espacio de 280 caracteres en el microblogging

La minería de datos es una herramienta muy utilizada y mucho más completa en la selección, clasificación y filtrado de mensajes en Twitter. Los métodos de data mining revelan esta información y la transforman en un valioso conocimiento tanto retrospectivo (histórico) como prospectivo (proyecciones) o comprensivo (entender lo que ocurre). Se distinguen tres tipos de minería: de textos, grafos y multimedia

El objetivo de la minería de datos es aprovechar la hiperabundancia de información, cabe destacar que las técnicas de lingüística de corpus, clustering o análisis de concordancia de textos automatizados y la detección de las polaridades forman parte de la minería de textos dentro de la minería de datos como proceso general de obtención de resultados.

El uso de la minería de textos para la detección de polaridad y opiniones es bastante efectivo, aunque está en constante mejoramiento en base a sus características lingüísticas y aún precisa de algoritmos más efectivos en la detección de recursos como la ironía y el sarcasmo aseveran autores como: Reyes et al. (2012), Ortega et al. (2013), Hernández (2017) y Van Hee (2017).

En la minería de textos se utilizan técnicas simbólicas que hacen uso de recursos léxicos para revisar la polaridad (Kwabla et al, 2017). La tarea de clasificar correctamente la polaridad de un mensaje como positivo, negativo o neutro (Sygkounas, E. Rizzo, G. Troncy, R., 2016), es una tarea compleja que aborda entre otros aspectos el manejo de la sintaxis y la semántica. En la sintaxis se debe tener en cuenta la forma cómo está escrito el mensaje, es decir, la estructura gramatical usada y si ésta incluye emoticonos, contracciones y elongaciones en las palabras.

Sin embargo, en la minería de textos se responde al filtrado de sentimientos en un soporte específico dentro del constructo simbólico de la muestra analizada: el texto escrito, sus características y cualidades. Sin embargo, ante las propiedades de las publicaciones del usuario en las redes sociales, no podemos eliminar en el contexto enfocado hacia la detección 
de categorías como las relaciones sociales y el contenido multimedia en la expresión. Aunque la mayoría de las investigaciones precedentes se orientan hacia la minería de textos existen pocas excepciones que le han prestado interés a las relaciones sociales y el contenido multimedia. En tal sentido autores como Welbers y Opgenhaffen (2019), Wu et al (2011), y Gruzd et al (2011) expresan una particular importancia de los enlaces sociales en la conversación en comunidades virtuales como factor determinante en la producción de sentido y los discursos. Los enlaces sociales son medibles a través de la minería de grafos, técnica que proporciona herramientas para mapear las estructuras de datos y para encontrar nuevas conexiones (relaciones) entre los objetos o nodos (Chakrabarti et al. 2004).

Además, Vásquez (2019) habla de un análisis multimodal en la relación semiótica entre texto del tweet y la imagen añadida, suscribimos que dicha relación supone una unidad de sentido. Estudiar no solo el texto escrito sino incluir valores multimodales es importante dado que no es en los datos estructurados en donde se encuentra el mayor volumen de información que existe al nivel mundial, sino en multimedia como es el caso de imágenes, texto, audio y vídeos (Oviedo y Velez, 2016:127).

Algunos autores (Dias, 2018; Nuñez y Fernández, 2018; Bouku y García, 2019; Oiza, 2019; Bronwyn, 2020; Mancera, 2020; Molpeceres y Filardo, 2020; Ozduzen y Macgarry, 2020) han utilizado recientemente el análisis del discurso multimodal para el estudio de producciones discursivas audiovisuales en Twitter, siendo esta una metodología cualitativa adaptada al microblogging, mostrándose un particular interés en el estudio de los memes. El análisis del discurso multimodal es un paradigma emergente en el campo de los estudios del discurso y la lingüística que amplía el estudio del lenguaje per se al estudio del lenguaje en combinación con otros recursos (O'Halloran, 2016). El análisis del discurso multimodal se caracteriza por la importancia de entender los discursos como prácticas discursivas, las cuales se realizan con intenciones particulares en contextos determinados en varias modalidades que son dinámicas y exigen una cierta flexibilidad metodológica (Cárcamo, 2018: 169).

Hemos visto entonces que el principal problema es la fragmentación del mensaje o unidad de sentido (tweet) atendiendo a su soporte, y la complejidad metodológica que supone analizar Twitter como un espacio multisoporte con enfoque social- interactivo. Los métodos para estudiar el contenido que circula en la web constituyen un llamativo campo de exploración, lo que estimula el interés por la experimentación con creativos planteamientos conceptuales, 
modelos y herramientas, que abren posibilidades para analizar los mensajes difundidos. Como apunta Gómez (2017), las ciencias sociales parecen haber entrado en una fase de reconstitución metodológica para poder dar cuenta de fenómenos emergentes, complejos y resbaladizos.

La propuesta metodológica denominada análisis del discurso asistido por minería de datos es una recopilación de técnicas y métodos emergentes para describir el discurso atendiendo a las categorías circulantes que le dan forma y sentido. Con una naturaleza multisoporte la misma permite detectar patrones replicables en discurso a través del uso de softwares o aplicaciones para formatos como el texto escrito y multimodal, así como las relaciones sociales y las correspondientes reacciones in situ.

El análisis de discurso asistido por minería de datos presenta una sincronía entre propuestas metodológicas de corte cualitativo como el análisis del discurso de Van Dijk, el análisis del discurso multimodal, el análisis de contenido y modelos cuantitativos como la minería de textos que incluye técnicas de lingüística de corpus, el clustering, el análisis de sentimientos y la minería de opiniones, así como la minería de grafos, su correspondiente análisis de relaciones sociales y el minado multimedia. investigación.

Se trata de una propuesta que puede ser explicada como una guía de enfoque mixto, contextual y multimetodlógica que, si bien obtiene sus resultados a partir de los patrones detectados por aplicaciones, precisa de un manejo manual para el filtrado objetivo de los resultados de la muestra, así como para la detección de relaciones sociales de interés en la comunidad virtual, para describir el contenido multisoporte que aún no es factible a través de softwares y para darle forma de acuerdo a los objetivos de la investigación. De este modo el investigador de manera manual conduce la búsqueda y facilita los indicadores para detectar los patrones de repetición y los procesos que la máquina debe hacer.

\section{Contexto situacional: \#vacunacoronavirus \#coronavirusvaccine}

El 30 de enero de 2020 el Comité de Emergencias de la Organización Mundial de la Salud (OMS) declara que el brote de COVID 19 constituye una emergencia de salud pública de importancia internacional. La enfermedad fue declarada pandemia global el 11 de marzo de 2020 (OMS, 2020a). 
La emergencia y difusión de la enfermedad del coronavirus, sobre todo en la etapa inicial de la pandemia, causó incertidumbre a nivel mundial tanto por su alta capacidad de contagio como por la inexistencia de vacuna o tratamientos paliativos (Sued y Cebral, 2020).

Ante el aislamiento social y el cambio de la vida en todos los aspectos la aparición de una vacuna contra el SARSCov-2 ofreció una esperanza para una posible normalidad. No obstante se produce una cierta reticencia a la vacunación sobre todo ante la muestra de una carrera acelerada que permitió obtener varios tipos de vacuna, que no producen una inmunidad esterilizante del organismo y que tienen un corto o aún no determinado período de duración. Cruz et al. (2020) habla de un esfuerzo de las entidades por hacer valer el criterio de que la vacunación debe ser una voluntad moral relacionada con la salud de la colectividad, aunque no constituye una responsabilidad jurídica. Verweij (2017) expone que no se puede obviar que se administra masivamente a personas sanas, por lo que sus riesgos deben ser muy inferiores a sus beneficios.

En este caso, lo observable es que, ante la situación sanitaria, el tiempo en el que usualmente se aprobaba y procesaba una vacuna en un período aproximado de 5 años según Cruz et al. (2020) disminuyó y se ajustó a las prácticas de emergencia a que nos expuso la pandemia. Harrison y Wu (2020) mencionan que sería necesario también limitar el excesivo optimismo en la vacuna como solución técnica y objetiva al problema de la prevención y la salud de las poblaciones, porque obstaculizan otras medidas que pueden ser política y moralmente más controvertidas y producir más equidad.

El tema de investigación resulta de actualidad pues analiza un caso de particular interés en el acontecer internacional ante la irrupción de la pandemia de COVID 19. La llegada de una vacuna frente al SARS-CoV-2 que pueda frenar la expansión de la infección se ha postulado como la respuesta definitiva según Cruz et al (2020). Los productos de los laboratorios Pfizer, Janssen Pharmaceuticals Companies of Johnson \& Johnson, ModernaTX, AstraZéneca. SINOVAC, Gamaleya, entre otros han comenzado a aplicarse y distribuirse a largo plazo a la población, por lo que es pertinente el análisis del discurso sobre las vacunas asociado a los hashtags \#coronavirusvaccine y \#vacunacoronavirus en Twitter. El tema es una contribución al estudio de las opiniones publicadas sobre la vacuna contra el coronavirus y el análisis del discurso desde la comprensión y adecuación de metodologías en el espacio del microblogging. 


\section{Metodología}

Problema de investigación: ¿Cuál fue la representación simbólica generalizada en el discurso asociado al hashtag \#vacunacoronavirus y \#coronavirusvaccine?

La presente investigación fue aplicada a una muestra de 1 millón de tweets únicos 1 millón de tweets, de los cuales 600 mil correspondían a la etiqueta \#coronavirusvaccine y unos 400 mil con el hashtag \#vacunacoronavirus. Fueron publicados en la última semana del año 2020 (desde el 24 al 31 de diciembre) y sustraídos con las aplicaciones Stela y Brand24.

El objetivo principal fue caracterizar el discurso asociado al hashtag \#vacunacoronavirusy \#coronavirusvaccine. A partir de un enfoque mixto presentamos un reporte de tipo descriptivo, basado en análisis del discurso asistido por minería de datos.

Partimos de la premisa que la representación simbólica emitida en Twitter con los hashtags \#coronavirusvaccine y \#vacunacoronavirus está marcada por prácticas discursivas multisoportes generadas a través de categorías contextuales propias de los códigos de la interfaz del microblogging que dan lugar a ejercicios de polaridad y opinión sobre el acontecimiento noticioso.

Además de las dinámicas teórico metodológicas mencionadas anteriormente el aspecto cualitativo y manual sobre el objeto del discurso, en su forma textual, no ha sido suplantado sigue siendo como lo menciona Wodak y Meyer (2003) la realización del análisis fino, una técnica muy efectiva que se centra en el contexto, la superficie textual y los instrumentos retóricos, aunque para ello los softwares y herramientas digitales faciliten la obtención de resultados mejor acabados. Por tanto, aplicamos la siguiente:

Guía de análisis fino:

1. Regiones de la que se emite (de acuerdo a la descripción geográfica del usuario)

2. Porciento de tweets con enlaces.

3. Polaridad del total de los tweets por el tono discursivo

\footnotetext{
IICom (Instituto de Investigaciones en Comunicación)

Facultad de Periodismo y Comunicación Social

Universidad Nacional de La Plata
} 
4. Encuadres y su polaridad.

5. Recursos expresivos lingüísticos utilizados.

6. Grafos textuales más usados en la muestra

7. Usuarios con mayor influencia (por medición de grado de entrada, por centralidad de grado de eigenvector)

8. Hilo de los tweets con mayor tendencia a la conversación.

9. Recursos multimodales como forma de expresión discursiva en la muestra.

Resultados:

1. Regiones de las que se emite (de acuerdo a la descripción geográfica del usuario)

\#vacunacoronavirus

En este orden los países con más tweets de la muestra en español: España, Argentina, México, República Dominicana, Ecuador, Colombia, Nicaragua, Venezuela, Perú, Estados Unidos (Miami, Florida,Dallas y Texas), Rusia (Moscú).

\#coronavirusvaccine

En este orden los países con más tweets de la muestra en inglés: USA, India, Reino Unido, Canadá, Alemania, Emiratos Árabes Unidos (Qatar, Abu Dabi), Australia, Bélgica, Nepal, Kenia, Nigeria, Chipre, Francia, Tailandia, Islas Vírgenes Británicas, Indonesia, Sri Lanka, Pakistán, Corea del Sur, Guyana, Brazil, Irlanda, Singapur, Holanda, Suiza, España, Italia, República Checa, Irán, Sudáfrica, Turquía, Japón, Hungría, Egipto, Paraguay, Ghana, Bangladés.

2. Porciento de tweets con enlaces.

El $86 \%$ de los tweets contienen enlaces. Este contenido mayoritariamente redirige a noticias de medios de comunicación y la mayor parte de los temas se asocian a 
la logística para la vacunación, nuevas cepas o aplicación de la vacuna a figuras públicas.

3. Polaridad del total de los tweets por el tono discursivo

\section{Gráfico 1. Polaridad del total de tweets de acuerdo al tono del mensaje}

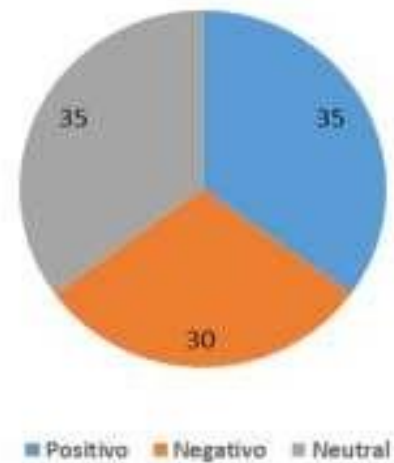

Fuente: Elaboración propia basada en resultados del software Stela

4. Encuadres y su polaridad 
Gráfico 2. Polaridad de acuerdo a los encuadres discursivos

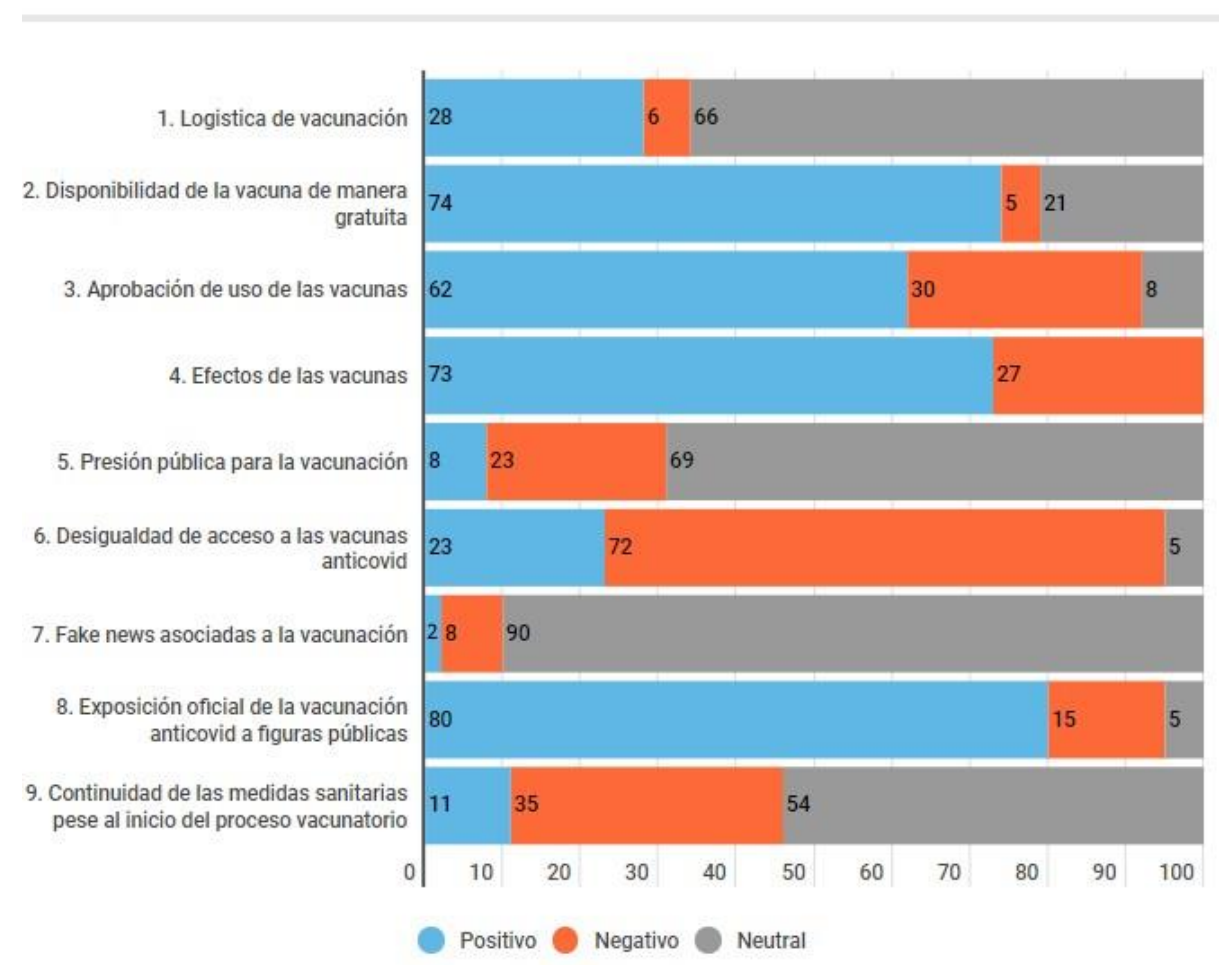

Fuente: Elaboración propia basada en resultados del software Stela a través de clustering.

\section{Recursos expresivos lingüísticos utilizados}

Los recursos del lenguaje más utilizados fueron el empleo de oraciones afirmativas en la distribución del contenido de los medios. Uso de macroestructuras semánticas organizadas en los hashtags. Utilización de enlaces en la mayoría de los casos para la continuidad o maximización del contenido emitido. Empleo de signos de exclamación y comillas para eufemismos, sentido irónico y para citas textuales referentes a discursos emitidos públicamente por personalidades políticas, del sector sanitario o periodistas. Uso de emojis para complementar y graficar, entre ellos destaca el emoticón de la vacuna, la señal de emergencia o la imagen de profesional sanitario. Se resaltan además los titulares entre corchetes cuando la expresión es algo ambigua en su contenido, se utiliza la mayúscula para resaltar frases. Hay 
una tendencia en acortar palabras para aprovechar el espacio de 280 caracteres como por ejemplo en inglés fwd para exponer forward.

Es usual el empleo de ciertos recursos del argot popular como cruzar los dedos, dios nos ayude, o el empleo de recursos como la masa dormida denota respectivamente una intención espiritual sobre la efectividad del asunto y cierta desconfianza en el logro de los resultados de los planes vacunación.

Se utilizan recursos expresivos (silogismos, metáforas, concatenación, anadiplosis, hipérbaton, etc.) y referentes sensacionalistas que tienden a causar incertidumbre y pánico respecto al fármaco, se emplean ciertos slogans para microargumentar situaciones puntuales. Así, por ejemplo:

- Posiciones positivas:

La vacuna, iqué gran idea!

Las buenas noticias sobre la \#VacunaDelCoronavirus se están contagiando .....jAhora todo el mundo la quiere! (HIPÉRBOLE)

Hoy es el principio del fin de la pandemia (HIPERBATON)

- Posiciones negativas:

Los países ricos distribuyen vacunas y los pobres esperan. (CONCATENACIÓN)

Hay una carrera entre virus y vacunas, y las vacunas no llegan a la velocidad necesaria. (ANADIPLOSIS)

La palabra de principio de 2021 será: Vacuna. La de final de este año será: Fracaso. (CONCATENACIÓN. SILOGISMO: las dos frases dan lugar a una tercera de manera implícita relacionada con la visión del usuario del fracaso de la vacuna).

La \#vacunación no los hará libres. El \#cumplimiento no devolverá la normalidad. Cuando la masa dormida se dé cuenta de esto puede que cambie de opinión. (SILOGISMO) ...más miedo a la vacuna que al virus. (COMPARACIÓN). 
Este proceso es un maratón, no una carrera, así que por favor continúe siguiendo el distanciamiento social actual. (METÁFORA)

5. Grafos textuales más usados en la muestra

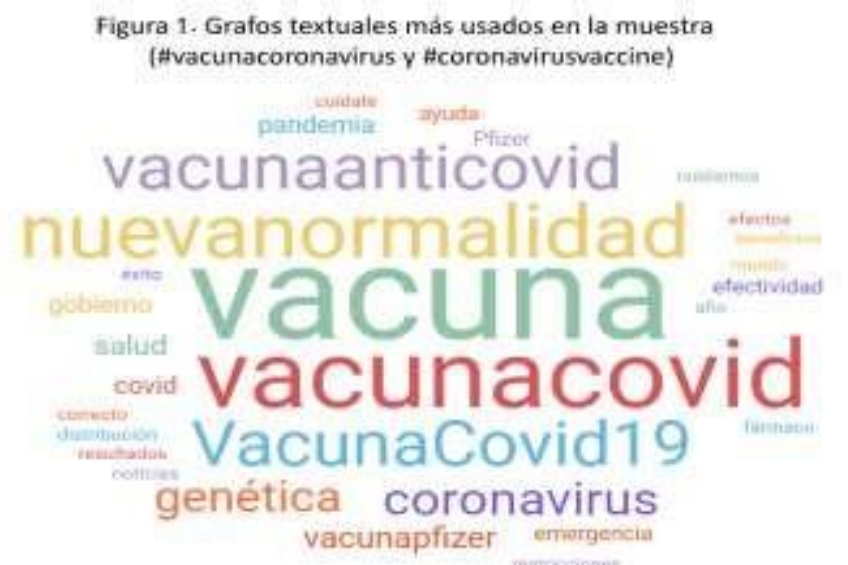

Fuente: Elaboración propia basada en los resultados del software Stela

6. Usuarios con mayor influencia (por medición de grado de entrada y por centralidad de grado)

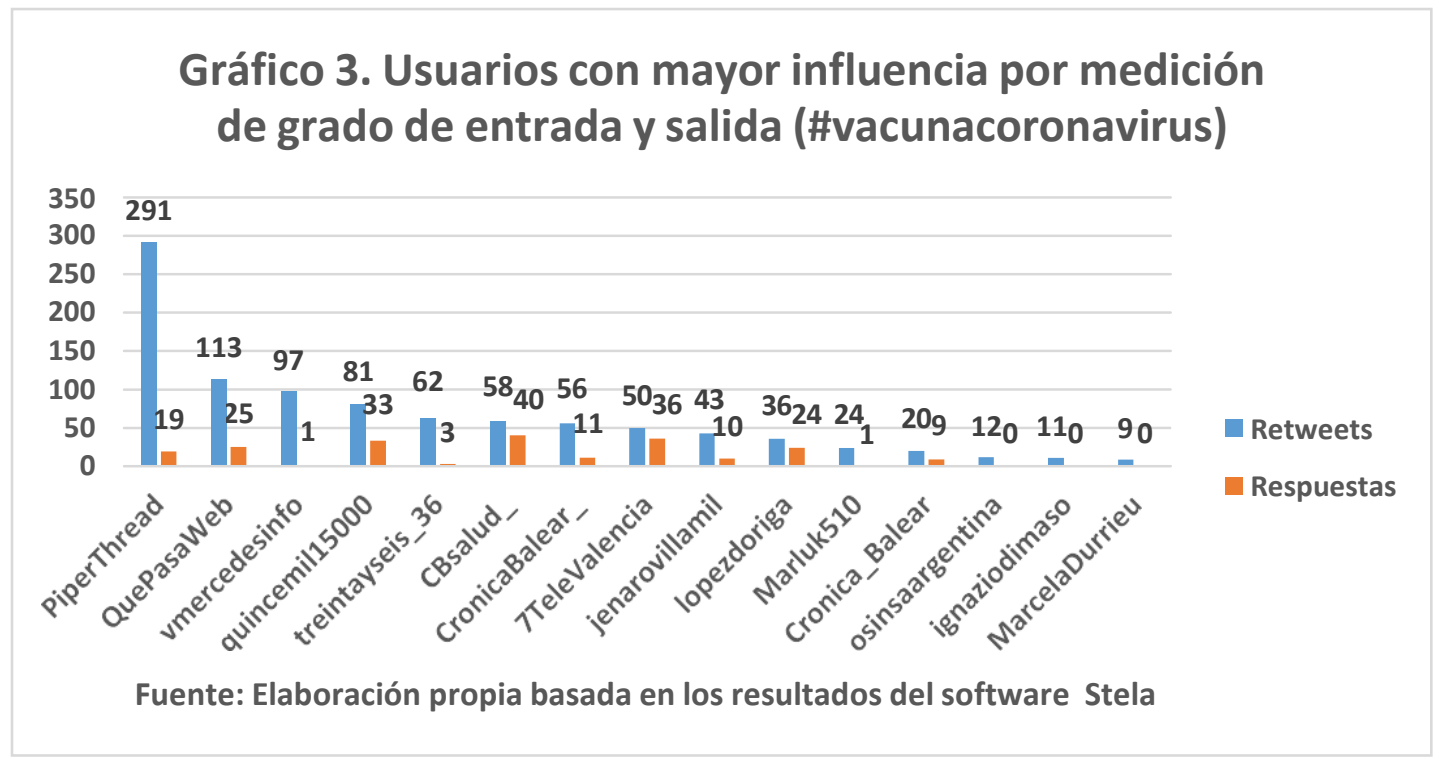



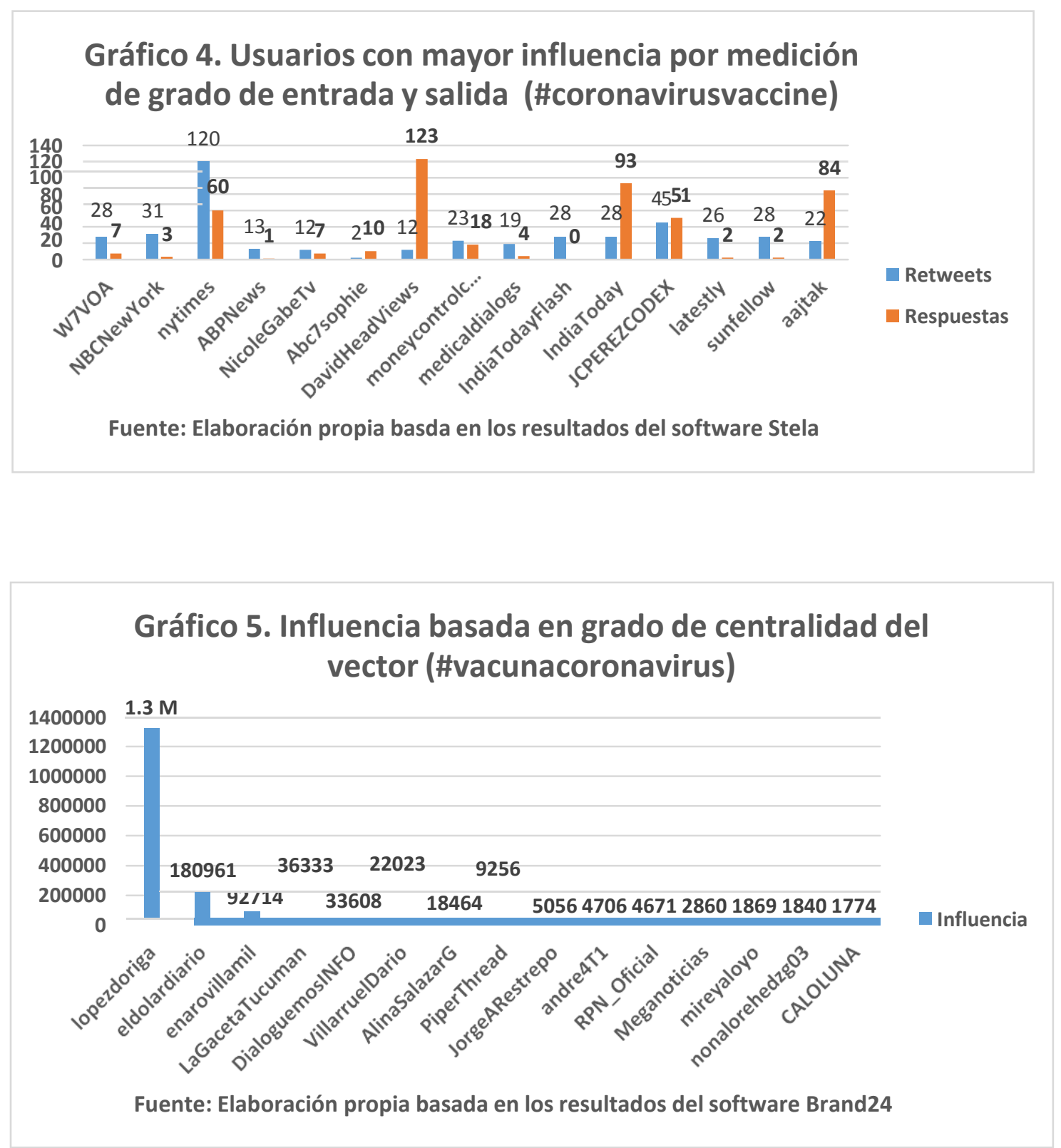

IICom (Instituto de Investigaciones en Comunicación)

Facultad de Periodismo y Comunicación Social

Universidad Nacional de La Plata 


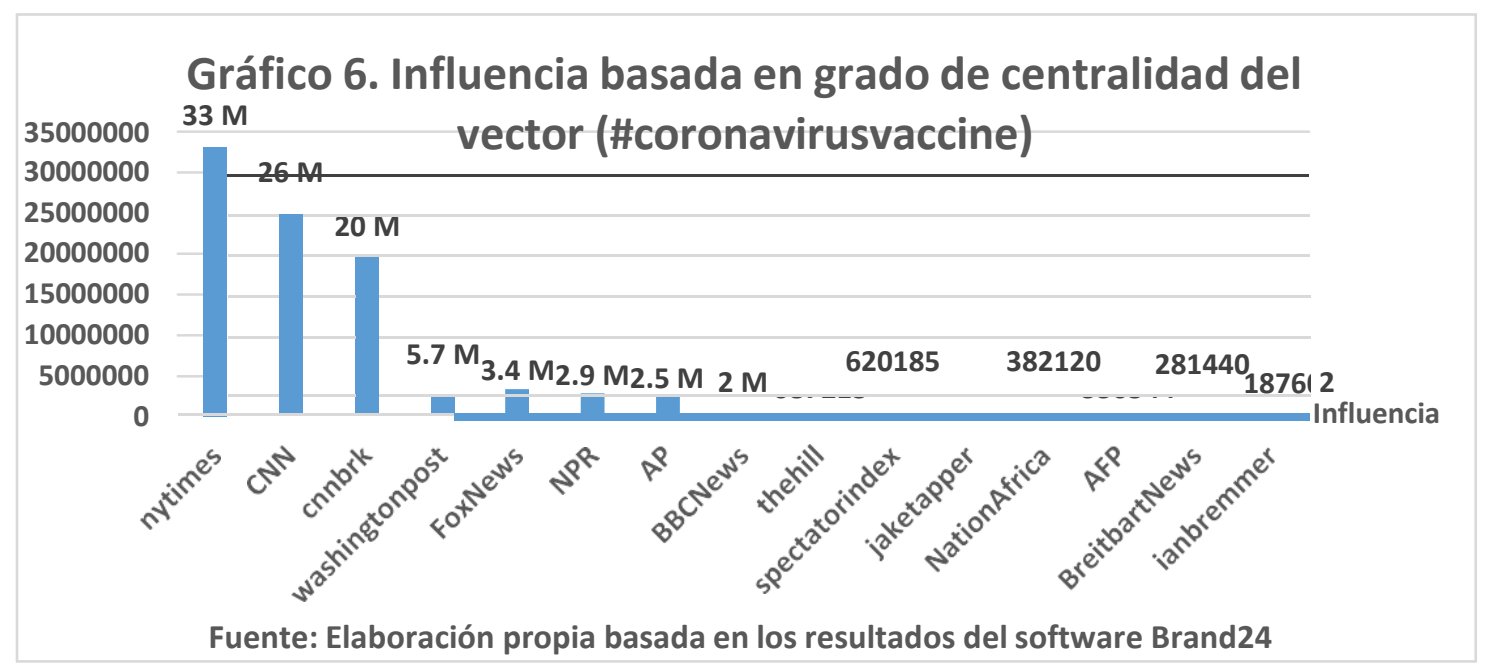

\section{Hilo de los tweets con mayor tendencia a la conversación}

Los tweets con mayor tendencia a la conversación son usuarios comunes o figuras públicas, como periodistas, que llaman a la acción con una pregunta o mensaje. En la muestra los medios de comunicación oficiales no producen esa retroalimentación. Los temas más tratados en los hilos fueron la incompetencia de implementación de la vacuna, la desigualdad de vacunación por un lado asociada a la cantidad de vacunas disponibles con respecto a la población de regiones y países, además las dudas e incertidumbre ante los efectos del fármaco.

\section{Recursos multimodales como forma de expresión discursiva en la muestra}

La multimodalidad en la muestra se aplica en primer lugar relacionada al texto rey con el uso de emojis para graficar el contenido expresado sin suplantarlo. No obstante, tal cualidad es más visible en los contenidos adjuntos al texto rey en el mismo tweet. En este caso como menciona Barthes (1977) hace función de anclaje e ilustración, es decir: en la mayoría de los casos complementa la información expresada de manera escrita graficando y connotando el encuadre tratado y en otros pocos casos la imagen o video es el centro del sentido expuesto en el tweet, así por ejemplo se hace referencia al año que inicia (2021) refiriéndose a las 
mutaciones del virus y en otro tweet donde la imagen indica el contenido del documento a firmar para obtener la vacuna que prácticamente exime de responsabilidades y revela que el medicamento está aún siendo evaluado. En los productos multimediales distribuidos suele mostrarse una forma de contar el proceso a través de imágenes y videos de usuarios comunes o figuras públicas como el momento de recibir la vacuna y los efectos posteriores, así mismo se reflejan las colas en Estados Unidos de América para obtener turnos de vacunación. Suelen mostrarse los datos gráficos compartidos tanto de la mutación del virus, de los reportes de casos positivos, porciento de mortalidad por número de casos y del funcionamiento de la inmunidad de rebaño por vacunación. Simbólicamente se muestra una imagen de las compras de navidad de 1957 en la que se aglomeran personas para denotar las modificaciones en la socialización por las medidas durante la pandemia.

\section{Conclusiones}

El discurso emitido en Twitter bajo los hashtags \#vacunacoronavirus y \#coronavirusvaccine expresa la incertidumbre general con respecto a la vacunación anticovid a finales del año 2020. Los medios de comunicación en el período analizado se dedicaron a distribuir noticias asociadas fundamentalmente a la logística del proceso, los cambios mutacionales del virus y la efectividad de las vacunas. Sin embargo, las conversaciones en la plataforma tienden a resaltar encuadres asociados a la desigualdad de vacunación en varios aspectos, los efectos adversos de las vacunas, el miedo a las posibles implicaciones a largo plazo y una discusión alternativa sobre las decisiones organizativas y la posible solución no neutralizante del fármaco.

Hay que resaltar el amplio número de tweets relacionado fundamentalmente con la desigualdad de vacunación y las oportunidades de unos países con respecto a otros para acceder y producir el fármaco. No obstante, no se puede dejar de lado que, aunque constituyen minoría, hay un índice de esperanza en los mensajes.

Los recursos multimodales completan el contenido del texto rey en las publicaciones graficando la información que, si por un lado reproducen un storytelling de casos particulares y regionalizados, también muestran avances del proceso y de los cambios mutacionales del 
virus. El uso de emojis y hashtags complementa la naturaleza contextual del discurso en la plataforma y los modos y modas de la comunicación contemporánea.

Destaca la influencia social de usuarios no vinculados a instituciones mediáticas, las imágenes que narran sucesos particulares para la distribución y aplicación de vacunas. Los usuarios con mayor grado de entrada y grado de centralidad se estructuran como centro de la conversación sugiriendo o proyectando una influencia sobre las discusiones conversacionales que tuvieron lugar en los hilos de la muestra.

Los enfoques literarios y los recursos expresivos se utilizan para llamar la atención en los textos cortos, incluso empleando estrategias basadas en el uso de mayúsculas, signos de exclamación, algunas encuestas, entre otros. El principal cambio insertado en la discusión general es sobre todo la continuidad de la distancia entre personas, evitar aglomeraciones y el uso de mascarilla como modo de prevención pese a la aplicación vacunal. La utilidad del análisis del discurso asistido por minería de datos en este caso permitió sustraer la información, clasificarla y llegar a conclusiones sobre los estados de opinión respecto a las vacunas anti-covid19 en los diferentes formatos y el estilo del microblogging.

\section{Referencias bibliografícas}

Abascal, R.; López, E. y Zepeda, S. (2014). Detección de patrones y grupos de sentimientos a partir del análisis de tuits políticos. Pistas Educativas, 35(108), 1672-1691. https://bit.ly/2VWUAtN

Arcila, C.; Ortega, F.; Jiménez, J. y Trullenque, S. (2017). Análisis supervisado de sentimientos políticos

en español: clasificación en tiempo real de tweets basada en aprendizaje automático. El profesional de la información, 26(5), 973-982. https://bit.ly/2AR9N7H

Barthes, R. (1977). The Rhetoric of the Image. En S. Heath (ed.), Image-Music-Text (pp. 3251). London: Fontana. 
Cárcamo, B. (2018). El análisis del discurso multimodal: una comparación de propuestas metodológicas. Forma y función, 31 (2), 145-174. https://bit.ly/3rkJcV3

Chakrabarti D.; Papadimitriou S.; Modha,D. \& Faloutsos, C. (2004). Fully automatic crossassociations. ACM SIGKDD, 79-88. https://bit.ly/2WjowiJ

Conde, M.A.; Pullaguari, K. P. y Prada, O.A. (2019). Comparativa de la competencia mediática en comunicación política vía Twitter durante las campañas electorales de candidatos presidenciales en España, Ecuador y Colombia. Contratexto, (32), 41-70. http://doi.org/dx78

Cruz, M., Hortal, J., y Padilla, J. (2020). Vísteme despacio que tengo prisa. Un análisis ético de la vacuna del COVID-19: fabricación, distribución y reticencia. Enrahonar. An International Journal of Theoretical and Practical Reason, 65, 57-73. https://bit.ly/31/Jgcx

Dackow,C. (2020). La retórica Twittera y el Ethos polémico en la disputa por la legalización del aborto en Argentina. https://bit.ly/3d5c3aZ

Du, Y., Masood, M. A., \& Joseph, K. (2020). Understanding visual memes: An empirical analysis of text superimposed on memes shared on twitter. In Proceedings of the International AAAI Conference on Web and Social Media (Vol. 14, pp. 153-164).

Engesser, S. y Humprecht, E. (2015). Frecuency or skillfulnes. Journalism studies, 16(4), 513529.http://doi.org/ggb592

Ferreira, R. M. (2019). Classificação automática de discursos de ódio em textos do Twitter . Tesis de Diploma. Brasil.

Figueira, Á. \& Guimarães, N. (2017). Detecting Journalistic Relevance on Social Media: A twocase study using automatic surrogate features. In Proceedings of the 2017 IEEE/ACM International Conference on Advances in Social Networks Analysis and Mining 2017 (pp. 11361139). http://doi.org/dx79

García, J.G.; Henríquez, P. y Herrera, J. (2019). Analítica de Twitter para el estudio de las emociones primarias durante el terremoto de México 2017. Revista Ibérica de Sistemas e Tecnologías de Informção, (E19), 479-492. https://bit.ly/3fc1z9c 
Gindin, I., Castros, S., Coiutti,N., Cardoso, A. y Rostagno, J. (2019). Emoción en acción. El caso de \#RosarioSangra en Twitter (Rosario, Argentina, 2016). Ámbitos. Revista Internacional de Comunicación 43(1), 48-69. doi: 10.12795/Ambitos.2019.i43.03.

Gómez, E. (2017). Etnografía celular: una propuesta emergente de etnografía digital, Virtualis, 8 (16), pp. 77-98. https://bit.ly/3p2oeuK

Gómez, M.E. (2014). Manual imperfecto para políticos en tiempo irreal. Universidad de La Habana, Cuba.

González, J.A.; Lluís, F.; Ferran, P. (2018). Análisis de sentimientos en Twitter basado en aprendizaje profundo. ELiRF-UPV at TASS 2018. https://bit.ly/34vpywu

Gruzd, A.; Wellman, B. \& Takhteyev, Y. (2011). Imagining Twitter as an imagined community. American Behavioral Scientist, 55(10), 1294-1318. http://doi.org/dhbc34

Gutiérrez, V. (2016). Retórica de los discursos digitales. Una propuesta metodológica para el análisis de los discursos en Twitter. Aposta. Revista de Ciencias Sociales, (69), 67-103. https://bit.ly/3vCfuOo

Harrison, E.A. y Wu, J.W. (2020). «Vaccine confidence in the time of COVID-19». European Journal Epidemiology, 35, 325-330. https://doi.org/10.1007/s10654-020-00634-3

Hernández, D. I. (2017). Irony and sarcasm detection in Twitter: The role of affective content. Universidad de Valencia, España. https://bit.ly/2MO5Mno

Hootsuite \& We are social (2021). The world's most-used social platforms. https://bit.ly/3vSinLw Kwabla, S.; Kwame, N. \& Katsriku, F. (2017). Sentiment Analysis of Twitter Feeds using Machine Learning, Effect of Feature Hash Bit Size. Communications on Applied Electronics (CAE), 6(9), 16-21. http://doi.org/dx8d

Mancera, A. (2020). Estudio exploratorio de las estrategias de encuadre discursivo en memes humorísticos publicados en Twitter durante las elecciones generales de noviembre de 2019 celebradas en España. Revista Dígitos, 1(6), 197-217. http://10.7203/rd.v1i6.180 
Manovich, L. (2002). The language of new media. MIT press.

Marín, A.F y Quintero, J.M. (2018). Confianza en el proceso de paz en Colombia en Twitter. Revista Mexicana de sociología, 80(1), 115-137. https://bit.ly/3i4H5DL

Martínez, J.J. (2017). Minería de opiniones mediante análisis de sentimientos y extracción de conceptos en Twitter. Universidad Complutense de Madrid, España. https://bit.ly/2UtIWac

Méndez, M. C. (2020). La construcción de la identidad lingüística de Santiago Abascal en Twitter. Revista Estudios del Discurso Digital (REDD), (3), 52-79.

https://doi.org/10.24197/redd.3.2020.50-77

Molpeceres, S.y Filardo, L. (2020). Llamamientos feministas en Twitter: ideología, identidad colectiva y reenmarcado de símbolos en la huelga del $8 \mathrm{M}$ y la manifestación contra la sentencia de 'La Manada'. Revista Dígitos, 1(6), 55-78. https://bit.ly/3fy0Ce8

Moya, M. y Herrera, S. (2015) Cómo puede contribuir Twitter a una comunicación política más avanzada. Arbor, 191(774). http://doi.org/dx8g

Nuñez, S., y Fernández, D. (2018). Discurso visual posmachista en Twitter: análisis multimodal de la iconicidad de la victimización. Revista Dígitos, (4), 131-149. https://bit.ly/3wOCU3j

O’Halloran, K.L. (2016). Análisis del discurso multimodal. Revista latinoamericana de estudios del discurso, 12(1), 75-97. https://bit.ly/3fQwbyU

Olave, G. (2019). Combates verbales en Twitter. El caso de la expresidenta argentina Fernández de Kirchner en las elecciones legislativas de 2017. Cultura, lenguaje y representación: revista de estudios culturales de la Universitat Jaume I, 21, 113-132.

Ortega, A.; Gutiérrez, E. y Montoyo, A. (2013). SSA-UO: Unsupervised Twitter Sentiment Analysis Second Joint Conference on Lexical and Computational Semantics ( ${ }^{*}$ SEM). Seventh International Workshop on Semantic Evaluation, (2), 501-507. https://bit.ly/2SrWHUw

Oviedo, E.A.; Oviedo A. I. y Velez, G.L. (2016). Minería multimedia: hacia la construcción de una metodología y una herramienta de analítica de datos no estructurado. Revista Ingenierías, 16(31), 125-142. http://doi.org/dx8j

\footnotetext{
IICom (Instituto de Investigaciones en Comunicación) 
Ozduzen, O. y McGarry, A. (2020). Huellas digitales de las revoluciones de Twitter: resistencia, polarización y vigilancia a través de imágenes y textos controvertidos de Occupy Gezi. Revista Internacional de Comunicación , 14 , 21. https://bit.ly/3wJp3en

Padilla Herrada, M. S. (2015). La argumentación política en twitter. Discurso \& Sociedad, 9 (4), 419-444. https://bit.ly/3yNbH2F

Percastre, S., Pont, C., y Codina, LI. (2017). Propuesta de diseño muestral para el análisis de Twitter en comunicación política. El profesional de la información, v. 26, n. 4, pp. 579-588. https://doi.org/10.3145/epi.2017.jul.02

Percastre, S., Pont, C., y Suau, G. (2019). La gestión comunicativa en redes sociales digitales de la emergencia del Ébola en España. The communicative management in social media of the emergence of Ebola in Spain., 80-90. http://10.20318/recs.2019.4437

Pérez, A. (2019). Una propuesta metodológica para el análisis del discurso político en Twitter: el caso \#PUIGDEMONT. En Comunicación digital: retos y oportunidades. https://bit.ly/3wlH4cT

Pérez, E. (2017). Entre el decir y lo dicho. Dinámicas discursivas del nuevo periodismo electrónico, sobre procesos políticos de alto perfil de polarización, en el escenario mediático latinoamericano. Tesis Doctotal. Universidad de la Habana. Cuba.

Pérez, E.; Haber, Y.; Díaz, J. y Zamora, L. (2017). Un modelo periodístico. Interrelación teórica del Nuevo periodismo electrónico y la plataforma Twitter, en el escenario mediático latinoamericano. Perspectivas de la Comunicación, 10(1), 157-186. https://bit.ly/3aYBlyW

Pérez, S. I. (2013). Tecnologías digitales, análisis del discurso y multimodalidad : de la lingüística crítica a la semiótica social. Revista de ciencias sociales, 5(23), 29-47. http://ridaa.unq.edu.ar/handle/20.500.11807/1565

Pulido, M.; Hernández, V. y Lozano, A. (2021). Uso institucional de Twitter para combatir la infodemia causada por la crisis sanitaria de la Covid-19. Profesional de la información, v. 30, n. 1, e300119. https://doi.org/10.3145/epi.2021.ene.19 
Rebollo, C. y Gualda, E. (2017) La situación internacional de las personas refugiadas y su imagen en Twitter. Un reto para la intervención desde el Trabajo Social. https://bit.ly/3p5hWdH

Reyes, A. (2012). Linguistic-based Patterns for Figurative language Processing: The Case of Humor Recognition and Irony Detection. Universidad de Valencia, España.

https://bit.ly/3dRDZ1c

Reyes, J.A.; Paniagua, F. y Sánchez, L. (2017). Minería de opiniones centrada en tópicos usando textos cortos en español. Research in Computing Science, 134, 151-162.

http://doi.org/dx8m

Rodríguez, K. y Haber, Y. (2020). Análisis de sentimientos en Twitter aplicado al \#impeachment de Donald Trump. Revista Mediterránea de Comunicación/MediterraneanJournal of Communication, 11(2), 199-213. https://www.doi.org/10.14198/MEDCOM2020.11.2.23

Rodríguez, K., Haber, Y y Gómez, M.E. (2021). Humanidades digitales: un acercamiento a metodologías emergentes desde el caso \#electionsUSA. Revista Alcance, 10(25), 301-323. https://bit.ly/3w0Czer

Sharag, A., Ye, X., y Spitzberg, B. (2018). Multilevel model of meme diffusion of fracking through Twitter. Chinese Sociological Dialogue, 3(1), 17-43. http:// 10.1177/2397200917752646

Sued, G.E. y Cebral, M. (2020). Voces autorizadas en Twitter durante la pandemia de COVID19: actores, léxico y sentimientos como marco interpretativo para usuarios ordinarios. Revista de Comunicación y Salud,Vol. 10, no 2, 549-568. https://doi.org/10.35669/rcys.2020.10(2).549$\underline{568}$

Sygkounas, E., Rizzo, G. y Troncy, R. (2016). A replication study of the Top Performing System in SemEval Twitter sentiment analyses. En International Semantic Web Conference (págs. 204219). Springer, Cham. https://bit.ly/3fbM1FD

Towers, S., Afzal, S., Bernal, G., Bliss, N., Brown, S., Espinoza, B., Jackson, J., Judson-Garcia, J., Khan, M., Lin, M., Mamada, R., Moreno, V. M., Nazari, F., Okuneye, K., Ross, M. L., Rodriguez, C., Medlock, J., Ebert, D., \& Castillo-Chavez, C. (2015). Mass Media and the 
Contagion of Fear: The Case of Ebola in America. PLOS ONE, 10(6), e0129179. doi: 10.1371/journal.pone.0129179

Van Dijk, TA (2000). El discurso como interacción social. Editorial Gedisa. Barcelona.

Van Hee, C. (2017) Can machines sense irony? Universidad de Gante, Bélgica. https://bit.ly/2SIld6w

Vásquez, L.C. (2019). Semiosis entre palabras e imágenes en Twitter. ¿Cómo construyen significado los candidatos presidenciales de las elecciones 2017 de Chile? Galaxia, 42, 40-56. http://doi.org/dx8r

Vela, C., Cantamutto, L., y Núñez, M. (2021). Análisis del hashtag \#mequedoencasa: la conversación digital por Twitter ante la emergencia producida por la COVID-19. Íkala: Revista de Lenguaje y Cultura, 26(2). https://doi.org/10.17533/udea.ikala.v26n2a08

Ventura, A.S. (2016) Argumentación y discurso político en Twitter. Análisis de la campaña presidencial argentina 2011. Aposta. Revista de Ciencias Sociales, 69, 39-66. https://bit.ly/3uDXO3s

Verbeke, M.; Berendt, B.; D’Haenens, L. \& Opgenhaffen, M. (2017). Critical news reading with Twitter? Exploring data-mining practices and their impact on societal discourse.

Communications, 42(2), 127-149. http://doi.org/dx8s

Verweij, M.F. (2017). «Ethics of Immunization». Reference Module in Biomedical Sciences, 710.

Vilares, D.; Alonso, M.A. y Gómez, C. (2013). Una aproximación supervisada para la minería de opiniones sobre tweets en español en base a conocimiento lingüístico. Procesamiento del Lenguaje natural, 51, 127-134. https://bit.ly/3c1Et3Y

Vilariño, D.; Zepeda, C.; Sanzón, Y.M.; Carballido, J.L.; Medina, C. y Flores, G. (2015) Aplicación del modelo Léxico-Sintáctico para detectar la polaridad de opiniones sobre profesores. Pistas Educativas, 36(113), 228-239. https://bit.ly/2KUtJs1 
Welbers, K. y Opgenhaffen, M. (2019). News through a social media filter. Different perspectives on immigration in news on website and social media formats. Journalism and Traslation in the Era of Convergence, 85-105. http://doi.org/dnvh

Wodak, R. y Meyer, M. (2003). Método de análisis crítico del discurso. Barcelona: Gedisa. [75] Wu, S.; Hofman, J.; Mason, W. \& Watts, D. (2011). Who Says What to Whom on Twitter. In Proceedings of the 20th International Conference on World Wide Web (pp. 705-714). http://doi.org/cxcibn

Wu, S., Hofman, J. M., Mason, W. A., \& Watts, D. J. (2011). Who says what to whom on twitter. In Proceedings of the 20th international conference on World wide web (pp. 705-714). https://bit.ly/3fsejfl

Zappavigna, M. (2011). Ambient affiliation: A linguistic Perspective on Twitter. New Media \& Society, 13(5), 788-806. http://doi.org/dx3px2 